\title{
An Image Processing Workflow to Quantify Penetration of Blob-like Structures into an Arbitrary Region of Interest
}

\author{
Trevor W. Lancon ${ }^{1}$
}

1. FEI Company, Visualization Sciences Group, Houston, TX, USA.

Automatic segmentation of blob-like structures is currently a prevalent challenge for many researchers. This effort is spearheaded primarily by life science applications due to the blob-like appearance of cells and nuclei in light microscopy datasets [1]. Approaches to this challenge range from segmenting directly by intensity to extracting semi-spherical objects with morphological operators. Researchers often desire to quantify various aspects of the detected objects describing their geometry, spatial distribution, and intensity statistics. Described here is a workflow that segments semi-spherical objects and quantifies their spatial distribution in relation to an arbitrary 3D reference geometry. A fluorescence microscopy dataset containing cellular structures penetrating a region-of-interest boundary illustrates this workflow using Amira 6.0.1 (FEI Company, Hillsboro, OR, USA).

Image denoising improves segmentation quality for a range of data types [2-4]. Appropriate denoising filters depend on the imaging modality. For this example we use Amira's despeckle filter to reduce impulse noise from the fluorescent signal. Mean background intensity is then subtracted from each voxel and negative voxels are truncated to zero. The contrast of local maxima is further enhanced with a difference of boxes filter to complete the image processing step.

Cell binarization begins with Otsu-based thresholding, which aims to minimize intensity variance within the resulting mask [5]. The watershed algorithm is then applied to separate erroneously connected spheres while preserving correctly conjoined objects (i.e. cells within telophase). Each cell is then assigned an index to retain its unique identity.

A distance map is calculated for the interior of the penetrable structure after the exterior is manually masked. The grayscale statistics within each object are then separately quantified using the distance map as intensity information. Numerous metrics describing cell shape and size are also calculable at this point.

Qualitative evaluation of the grayscale processing routine shows remarkable improvement in image quality (Figures 1A, 1B). Relevant cells are shown to be successfully thresholded and individually labelled (Figure 1C). Visually inspecting each cell represented as a point scaled to its mean distance from the penetrable boundary agree with the colorized distance map (Figure 1D). The resulting metrics describing cell morphology are also easily explored for statistically relevant correlations (Figure 2). Future work includes testing processing routines against ground truth segmentation and automating generation of the exterior mask.

[1] E Meijering, IEEE Signal Processing Magazine 29 (2012), p. 140.

[2] M Kalra et al, Radiology 228 (2003), p. 257.

[3] R Archibald et al, IEEE Transactions on Image Processing 13 (2004), p. 459.

[4] S Sulaiman and N Isa, IEEE Transactions on Consumer Electronics 56 (2010), p. 2702.

[5] N Otsu, IEEE Transactions on Systems, Man, and Cybernetics 9 (1979) p. 62. 


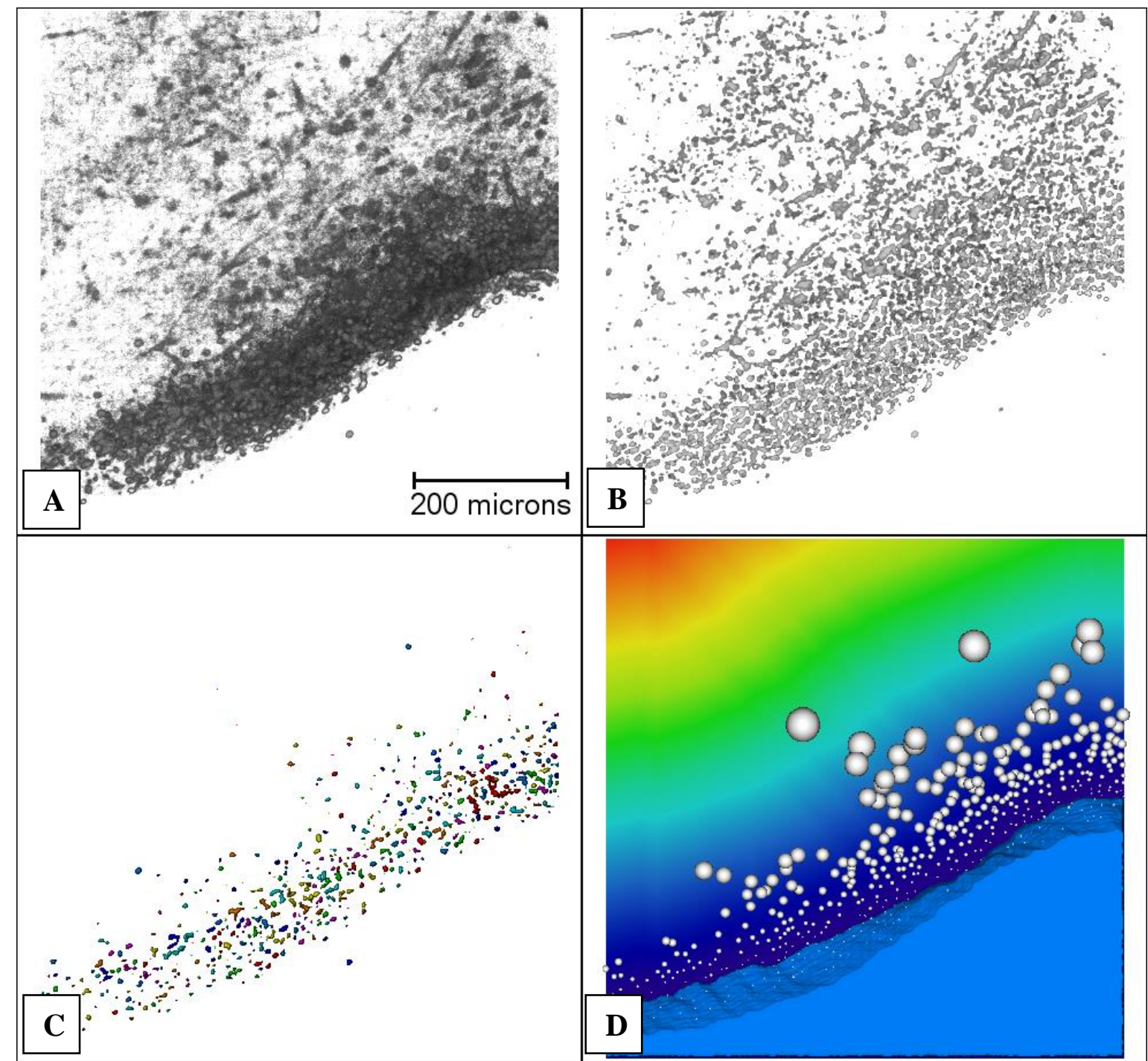

Figure 1. Shown here are (A) the original data before processing and (B) the processed dataset. Cells are shown (C) individually labeled after thresholding and (D) represented as points scaled to their mean distance from the boundary in context of the distance map. Scale bar is consistent in all images.
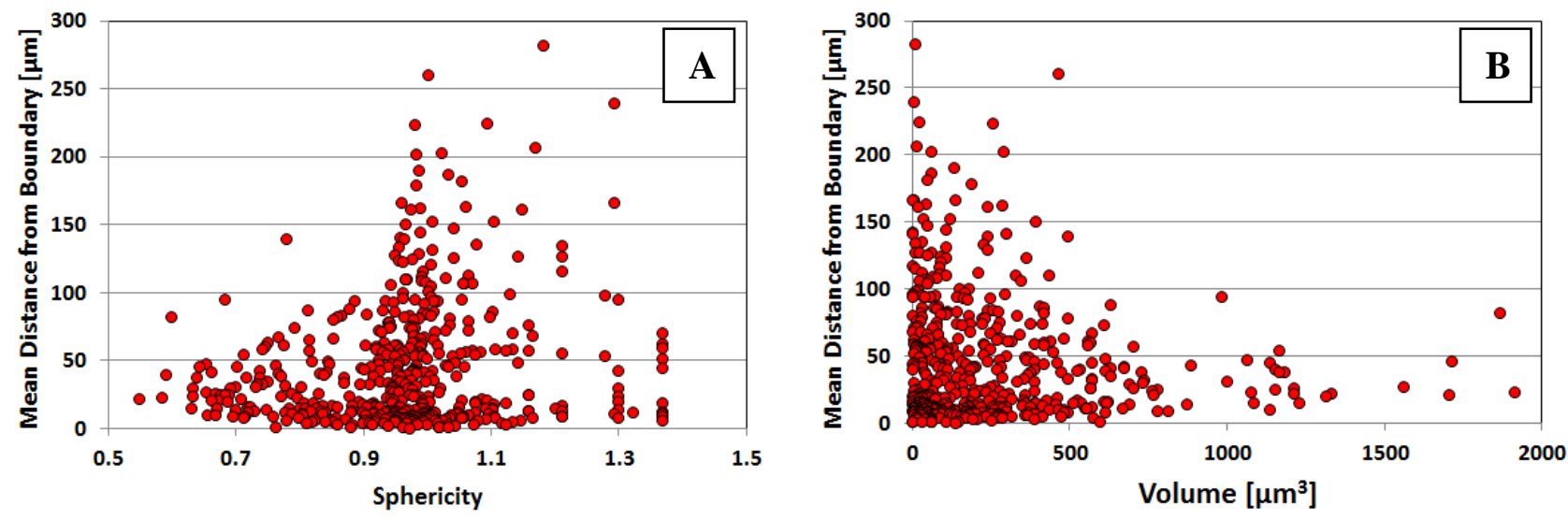

Figure 2. Examples of metrics describing cell behavior are mean cell penetrations through the boundary correlated to (A) sphericity and (B) volume. 